\title{
Cystathionine gamma lyase aggravates orthodontic root resorption in mice
}

\author{
Caizhu Lu, Liyuan Chen, Yongmei Hua \\ Department of Orthodontics, School of Stomatology/Shanghai Engineering Research Center of Tooth Restoration and Regeneration, Tongji \\ University, Shanghai 200072, China \\ Contributions: (I) Conception and design: C Lu, Y Hua; (II) Administrative support: Y Hua; (III) Provision of study materials or patients: All authors; \\ (IV) Collection and assembly of data: All authors; (V) Data analysis and interpretation: C Lu; (VI) Manuscript writing: All authors; (VII) Final \\ approval of manuscript: All authors. \\ Correspondence to: Yongmei Hua, Professor, DDS. Department of Orthodontics, School of Stomatology/Shanghai Engineering Research Center of \\ Tooth Restoration and Regeneration, Tongji University, 399 Middle Yanchang Road, Shanghai 200072, China. Email: yongmeihua@tongii.edu.cn.
}

Background: This study aimed to determine the contribution of cystathionine gamma lyase (CSE) to physiological and orthodontic root resorption in mice.

Methods: Mice genetically deficient in the $\mathrm{CSE}\left(\mathrm{CSE}^{-/}\right)$, the dominant hydrogen sulfide $\left(\mathrm{H}_{2} \mathrm{~S}\right)$ - generating enzyme in osteoclast were used in this study. Physiological and orthodontic root resorption was assessed with micro computed tomography (micro-CT) and scanning electron microscopy (SEM) in the mice at the age of 8-, 26-, and 52-week and in 8-week old mice following 1-, 2-, and 3-week orthodontic treatment, respectively. Hematoxylin and eosin (HE) and tartrate-resistant acid phosphatase (TRAP) staining were used for further determination of root resorption and the number of osteoclasts. The receptor activator of nuclear factor kappa-B ligand (RANKL)/osteoprotegerin (OPG) level in surrounding alveolar bone of the maxillary first molar after 2 -week orthodontic treatment was measured by reverse transcription polymerase chain reaction (RT-PCR).

Results: Root resorption lacunae (RRL) gradually and significantly increased with age in wild type (WT) and $\mathrm{CSE}^{-/-}$mice. The $\mathrm{CSE}^{-/-}$showed less RRL compared with the WT group. At each time point of orthodontic treatment, the $\mathrm{CSE}^{-/-}$group had less RRL and osteoclasts than the WT group. The orthodontically induced RANKL/OPG mRNA expression in the periodontal tissue in the $\mathrm{CSE}^{-/-}$group was lower than that in the WT group.

Conclusions: CSE contributes significantly to physiological and orthodontic root resorption.

Keywords: Root resorption; orthodontic tooth movement (OTM); cystathionine gamma lyase (CSE); receptor activator of nuclear factor kappa-B ligand (RANKL)/osteoprotegerin (OPG)

Submitted Jul 04, 2019. Accepted for publication Sep 11, 2019.

doi: $10.21037 /$ atm.2019.11.03

View this article at: http://dx.doi.org/10.21037/atm.2019.11.03

\section{Introduction}

Orthodontic tooth movement (OTM) is accompanied by periodontal tissue remodeling (1), which could improve masticatory ability and restore aesthetics $(2,3)$. However, there are many risks associated with this procedure. Root resorption is one of familiar complications during orthodontic treatment. Previous studies showed that over
$90 \%$ of orthodontic teeth would suffer from some degree of tooth resorption (4). It seems that root resorption is an unpredictable and unavoidable complication of orthodontic treatment (5). This undesirable result can reduce tooth stability, even cause permanent loss of the tooth. Various cells and cytokines are involved in the complicated process of orthodontic root resorption. Odontoclast with similar biological features to osteoclast $(6,7)$ has been considered 
to be responsible for the process of orthodontic root resorption (8). Antibody against receptor of macrophage colony-stimulating factor (M-CSF) blocked orthodontically induced osteoclastogenesis, odontoclastogenesis and root resorption (9-11). Osteoprotegerin (OPG)/RANK/ receptor activator of nuclear factor kappa-B ligand (RANKL) signaling pathway is known to play a crucial role in the differentiation of osteoclasts. OPG prevents RANK mediated activation of osteoclastic differentiation by binding to RANKL (12). Excessive orthodontic force induced bone resorption on the surface of the alveolar bone and increased OPG-positive cells and production of RANKL/RANK in osteoclasts and odontoclasts in the periodontal tissue, indicating OPG/RANK/RANKL might be involved in the process of root resorption, $(13,14)$.

Cystathionine gamma lyase (CSE) is a synthetase for hydrogen sulfide $\left(\mathrm{H}_{2} \mathrm{~S}\right)$, a gas transmitter produced by the somatic cell. $\mathrm{H}_{2} \mathrm{~S}$ contributes to a variety of pathophysiological processes in vivo (15). In recent years, many studies have shown that CSE mediates osteoclast activities $(16,17)$. Whether CSE- $\mathrm{H}_{2} \mathrm{~S}$ modulates the root resorption by regulating osteoclasts and odontoclasts remains unclear. The purpose of this study was to investigate the effect of CSE on root resorption as well as on OPG/RANKL expression under physiological condition and during orthodontic treatment in mice. The data might provide a useful reference for the treatment and prevention of orthodontic root resorption in clinical practice.

\section{Methods}

\section{Animals and OTM}

All animal procedures were in accordance with the Animal Management Regulations of the Ministry of Health of the People's Republic of China (Document No. 55, 2001) and approved by the Animal Use and Care Committee of Tongji University. CSE knock-out $\left(\mathrm{CSE}^{-/}\right)$mice were bred at the Shanghai Biomodel Organisms Center, Inc. 8, 26 and 52 weeks old male wild-type and $\mathrm{CSE}^{-/-}$mice were used to evaluate physiological root resorption. Each group has five animals. The orthodontic animal model was established on 8 -week-old male mice. Mice were housed under specific pathogen-free conditions with controlled temperature $\left(22 \pm 1^{\circ} \mathrm{C}\right)$, humidity $(40-60 \%)$ and a $12-\mathrm{h}$ light/dark cycle, and fed with soft food and water ad libitum.

To examine the effect of CSE on the orthodontic root resorption, an OTM mouse model was established as described in a previous study (15). Briefly, a nickel-titanium coiled spring $(0.2 \mathrm{~mm}$ in thickness, $1 \mathrm{~mm}$ in diameter and 10 $\mathrm{mm}$ in length, Smart Technology Co, Ltd., Beijing, China) was placed between maxillary incisor and first molar for 1, 2 and 3 weeks. The force produced was approximately $0.35 \mathrm{~N}$ (18) as measured by a dynamometer (HF-2; ALIYIOI). The right side without appliance was used as control.

\section{Micro-CT scanning}

The molars of mice were extracted from the maxillary bone carefully. The surrounding tissues were removed with tweezers and needles. The tooth was immersed in 2 percent sodium hypochlorite for about $20 \mathrm{~min}$. Root resorption craters were evaluated by micro-CT (SCANCO Medical AG, Bruttisellen, Switzerland) after the surface of the root surface was completely clear. The maxillary first molars were reconstructed by a self-contained $3 \mathrm{D}$ analysis software of micro-CT (SCANCO Medical AG).

\section{Electron microscope scanning}

The molars were further examined with a scanning electron microscopy (SEM) (TM-1000; Hitachi, Tokyo, Japan). The ratio of the root resorption area was calculated using ImageJ software (National Institutes of Health, Bethesda, Maryland, USA) (19). In addition, we assessed the root resorption by calculating the proportion of resorbed area on the pressure side of the distobuccal root of the maxillary first molar.

\section{Histological analysis}

Maxillae from all groups were dissected and prepared for histological analysis. The specimens were fixed in $4 \%$ paraformaldehyde at $4{ }^{\circ} \mathrm{C}$ for $24 \mathrm{~h}$, followed with decalcification $\left(4^{\circ} \mathrm{C}\right.$ for 4 weeks) by incubation with $10 \%$ ethylenediaminetetraacetic Acid (pH 7.4). The specimens were dehydrated in a series of alcohol baths beginning with $50 \%$ and progressing to $100 \%$. Thereafter, the samples were embedded in paraffin and five 4- $\mu \mathrm{m}$ horizontal sections were obtained at a depth of 100, 140,180, 220 and $260 \mu \mathrm{m}$ from the first molar bifurcation surface. and stained with hematoxylin and eosin (HE) and titrate-resistant acid phosphatase (TRAP) staining. We assessed the root resorption by the proportion of resorbed area on the pressure side of the distobuccal root of the maxillary first molar. 
Table 1 The primers used for real-time PCR

\begin{tabular}{lcc}
\hline Gene & Forward primers $\left(5^{\prime}-3^{\prime}\right)$ & Reverse primers $\left(5^{\prime}-3^{\prime}\right)$ \\
\hline RANKL & CAGAAGATGGCACTCACTGCA & CACCATCGCTTCTCTGCTCT \\
OPG & GTGGAATAGATGTCACCCTGTGT & TTTGGTCCCAGGCAAACTGT \\
GAPDH & AGCAGTCCCGTACACTGGCAAAC & TCTGTGGTGATGTAAATGTCCTCT \\
\hline
\end{tabular}

$\mathrm{PCR}$, polymerase chain reaction.

\section{$R N A$ extraction and reverse transcription polymerase chain reaction (RT-PCR)}

Total RNA from the alveolar bone surrounding the region of the first molar was extracted using TRIzol reagent (Invitrogen, USA) . RT-PCR Kit (Takara Bio, Inc., Otsu, Japan) was used to obtain complementary (c) DNA. Relative mRNA levels of OPG and RANKL were determined using a SYBR Green PCR Master mix and a LightCycler ${ }^{\circledR} 96$ Instrument (Roche, USA) following the manufacturer's protocol. The sequences for the RT-PCR primers used in this study are as follows: $O P G, 5$ '-GTGGAATAGATGTCACCCTGTGT3 ', 5 ' - TTT TGG T C C CA G GCAAACTGT - 3 '; RANKL, 5'- CAGAAGATGGCACTCACTGCA-3', 5'-CACCATCGCTTTCTCTGCTCT-3'; GAPDH, 5 ' - A GCA G T C C C GTA CA C T GG CAAA C - 3 ' , 5'-TCTGTGGTGATGTAAATGTCCTCT-3' (Table 1).

\section{Statistical analysis}

Data were expressed as mean \pm standard deviation. The statistical significance of differences among groups was calculated by independent 2-tailed Student's $t$-tests or oneway analysis of variance (ANOVA). $\mathrm{P}<0.05$ was considered significant.

\section{Results}

\section{CSE deficiency-reduced physiological root resorption (Figure 1)}

For the purpose of probing the function of CSE on physiological root resorption, we evaluated the root resorption in wide type (WT) and $\mathrm{CSE}^{-/-}$mice under physiological condition with micro-CT. Thirty (15 WT and $15 \mathrm{CSE}^{-/-}$) mice at the age of 8, 26 and 52 weeks were used to examine the physiological root resorption of the maxillary first molar. We found that no root resorption occurred in 8 weeks old $\mathrm{WT}$ and $\mathrm{CSE}^{-/-}$mice (Figure $1 A, D)$. Root resorption increased significantly in 26 weeks old mice (Figure 1B), and severe root resorption appeared at the age of 52 weeks in WT mice (Figure 1C). Root resorption was observed in 26 weeks old $\mathrm{CSE}^{-1-}$ mice (Figure $1 E$ ), and became more obvious at the age of 52 weeks (Figure $1 F$ ). It suggested that the root resorption increased with age under physiological condition and the CSE deficiency decreased root resorption in the 26- and 52-week old animals compared with WT group.

\section{CSE deficiency-reduced orthodontic root resorption assessed with micro-CT (Figure 2)}

In order to look into the effect of CSE on the orthodontic root resorption, we used micro-CT to assess the distobuccal root of the maxillary first molar of WT and $\mathrm{CSE}^{-/-}$with 1-, 2- and 3-week orthodontic treatment. We found root resorption lacunae (RRL) after loading orthodontic force for 1 week (Figure 2B,C). Root resorption became more obvious after loading for 2 weeks (Figure 2E,F) and was significantly aggravated at the end of 3 weeks loading (Figure 2H,I). At the same time point, root resorption in WT group was severer than that in $\mathrm{CSE}^{-/}$group. It suggested that the longer the orthodontic time, the more severe the root resorption is and knockout of the CSE gene can minimize the orthodontic root resorption.

\section{CSE deficiency reduced orthodontic root resorption determined by SEM (Figure 3)}

We also used the SEM to determine whether CSE deficiency altered the root resorption for maxillary first molar of the WT and $\mathrm{CSE}^{-/-}$mice following 1-, 2- and 3-week force application. We found that the SEM data showed a similar trend as the radiographic examinations above (Figure $3 A$ ). We further evaluated the root resorption index among the groups and found that the root resorption showed a trend of 

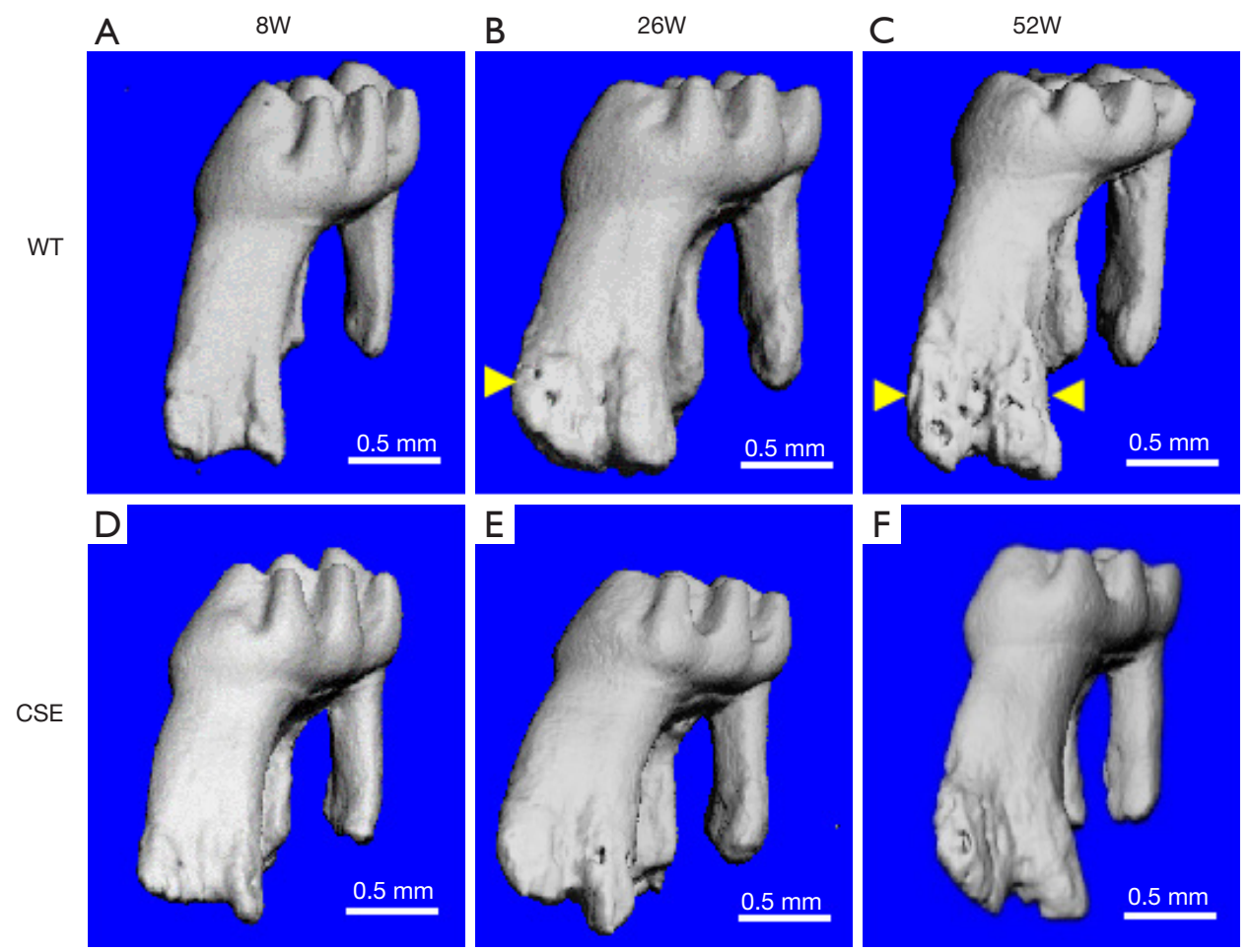

Figure 1 Effect of CSE on physiological root resorption. Reconstructed 3D micro-CT image showing root resorption of the maxillary first molar with age, (A) WT, 8W; (B) WT, 26W; (C) WT, 52W; (D) $\mathrm{CSE}^{-/}$, 8W; (E) CSE $\mathrm{CS}^{-/}, 26 \mathrm{~W}$; (F) CSE $\mathrm{CH}^{-/}$, $52 \mathrm{~W}$ group. Bar =0.5 mm. CSE, cystathionine gamma lyase; WT, wild type; W, week.

increase with orthodontic time, and the root resorption in $\mathrm{CSE}^{-/-}$mice was less than that in $\mathrm{WT}$ animals. The changes were statistically significant (Figure 3D).

\section{CSE deficiency reduced orthodontic root resorption confirmed with HE staining (Figure 4)}

Five horizontal sections of the maxillary first molars from each WT and $\mathrm{CSE}^{-/-}$animal with orthodontic treatment for 1, 2, and 3 weeks were assessed for the distobuccal RRL (Figure 4A). The root resorption index showed a trend of enhanced root resorption with the increase of orthodontic time, and the root resorption in $\mathrm{CSE}^{-/-}$mice was less compared with WT group, which was statistically significant (Figure 4C).

\section{CSE deficiency decreased orthodontically induced osteoclasts and $m R N A$ expression of RANKL/OPG (Figure 5)}

In this study, TRAP staining was performed to evaluate the role of CSE in root resorption and osteoclast response following 2-week orthodontic treatment. It was found CSE deficiency reduced orthodontic root resorption (Figure 5A) and osteoclasts. The changes were statistically significant (Figure 5C). The mRNA expression of RANKL/OPG in alveolar bone around the maxillary first molar was measured by RT-PCR (Figure 5D,E,F). The expression in $\mathrm{CSE}^{-1-}$ group was lower compared with WT mice.

\section{Discussion}

Root resorption is a physiological or pathological phenomenon of cementum and dentin defects (20). The incidence of orthodontic root resorption is extremely high. Root resorption can lead to an imbalance in the ratio of crown and root, reduction in root length, poor tooth stability in long term, even loss of tooth (21). On the pressure side of OTM, osteoclasts activated by many molecules in periodontal tissue cause the resorption of bone. Odontoclasts in cementum, as part of periodontal tissue, is also affected. Odontoclasts are very similar to osteoclasts. Under pressure, odontoclasts are activated 

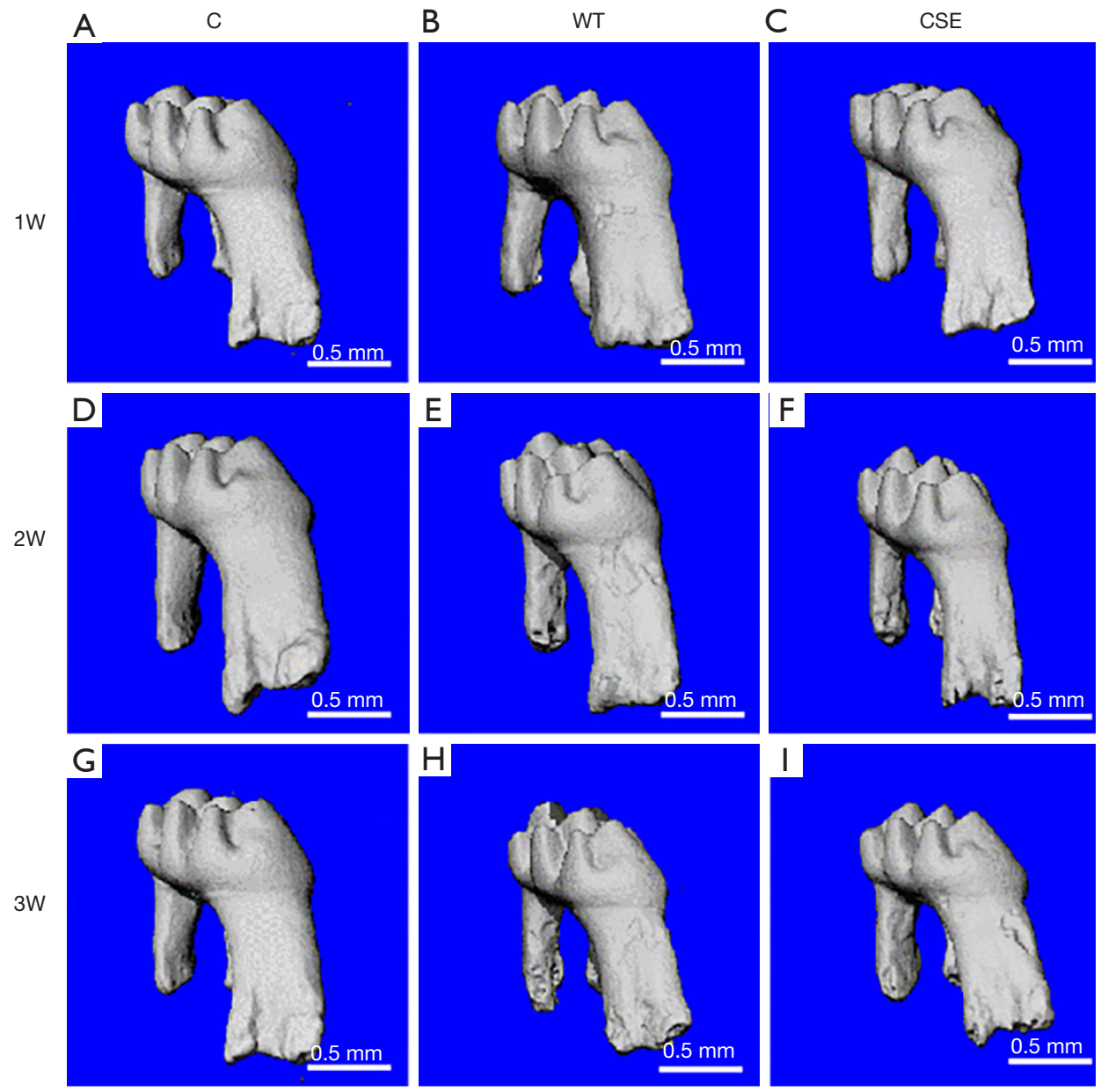

Figure 2 Effect of CSE on root resorption in orthodontic tooth movement model. Reconstructed 3D micro-CT image showing root resorption of the mesial surface of the distobuccal root of the maxillary first molar under orthodontic force, (A) control, 1W; (B)WT, 1W; (C) $\mathrm{CSE}^{-/-}, 1 \mathrm{~W}$; (D) control, 2W; (E) WT, 2W; (F) $\mathrm{CSE}^{-/-}, 2 \mathrm{~W}$; (G) control, 3W; (H) WT, 3W; (I) CSE ${ }^{-/}$, 3W group. CSE, cystathionine gamma lyase; WT, wild type; $\mathrm{C}$, control; $\mathrm{W}$, week.

to cause cementum resorption, which is similar to the process of alveolar bone resorption caused by osteoclasts. This phenomenon is considered as the orthodontic root resorption (22).

In this study, we found that the root resorption of maxillary first molars in both $\mathrm{CSE}^{-/-}$and WT mice was aggravated with age, suggesting that age might be a risk factor of root resorption. The possible causes include a decreased periodontal vascularity and inelasticity, thicker cementum and its firm attachment in the apical third of the root with increased susceptibility (23), Our data showed that $\mathrm{CSE}^{-/-}$mice has less age-related resorption compared with WT mice. CSE is a key enzyme that controls production of $\mathrm{H}_{2} \mathrm{~S}$ in the periodontal tissue. Our study indicates that
CSE $\mathrm{H}_{2} \mathrm{~S}$ might play a substantial role in physiological root resorption.

This study also demonstrated that the resorption area in both $\mathrm{CSE}^{-/-}$mice and WT mice increased with orthodontic time, indicating that the effects of orthodontics on root resorption is time-dependent. At each time point, the resorption area and the number of odontoclasts in $\mathrm{CSE}^{-/}$ group was less compared with the WT group, suggesting that CSE- $\mathrm{H}_{2} \mathrm{~S}$ plays a significant role in orthodontically induced root resorption caused by odontoclasts.

There are many factors that affect root resorption, such as sex, age, genetics, systemic factors, tooth type, root shape, and previous medical history. In addition, orthodontic treatment duration, applied force size, movement type and 

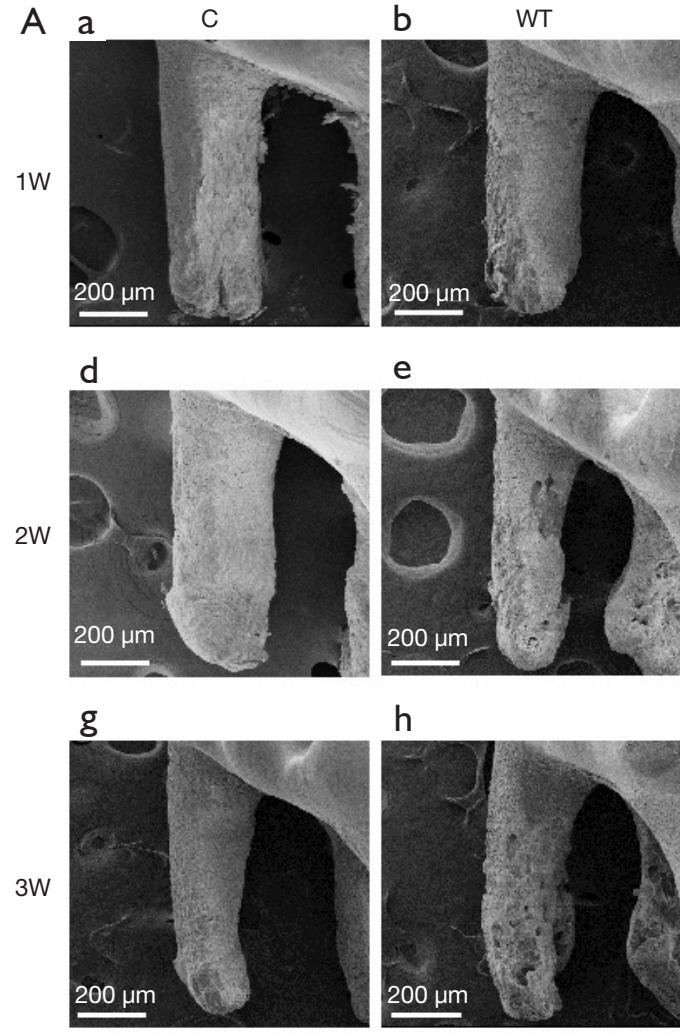

h
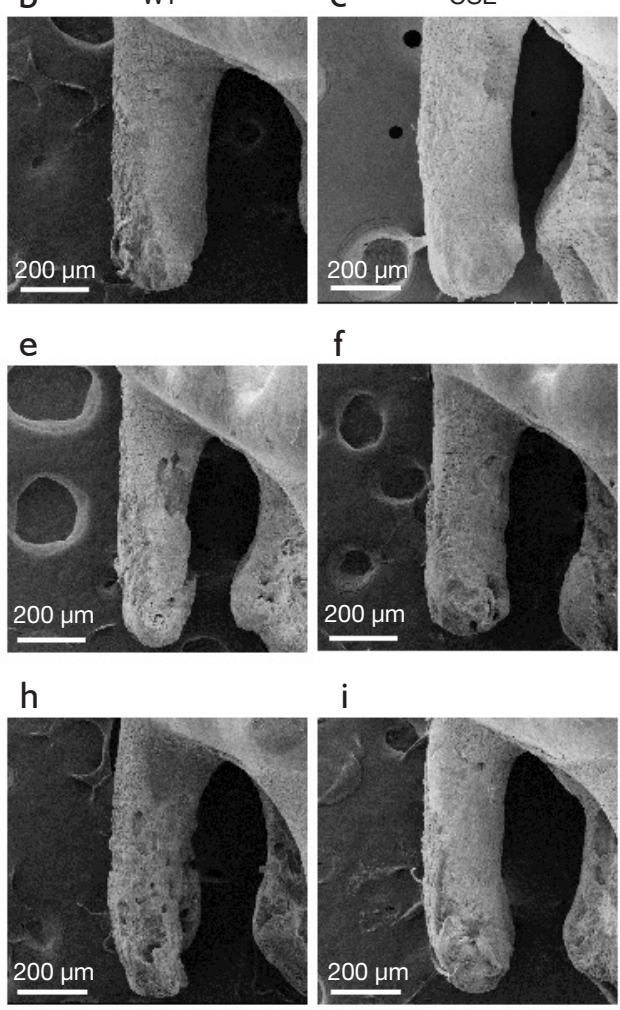

\section{$f$}

B

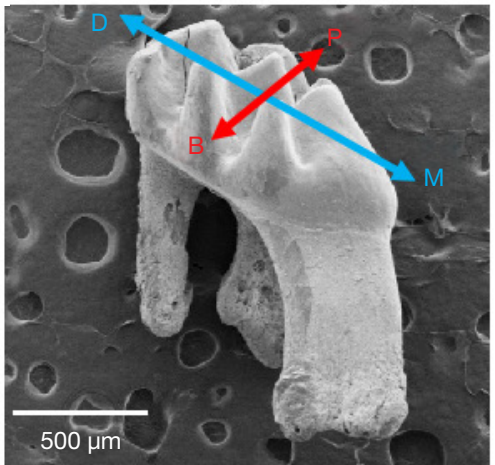

C

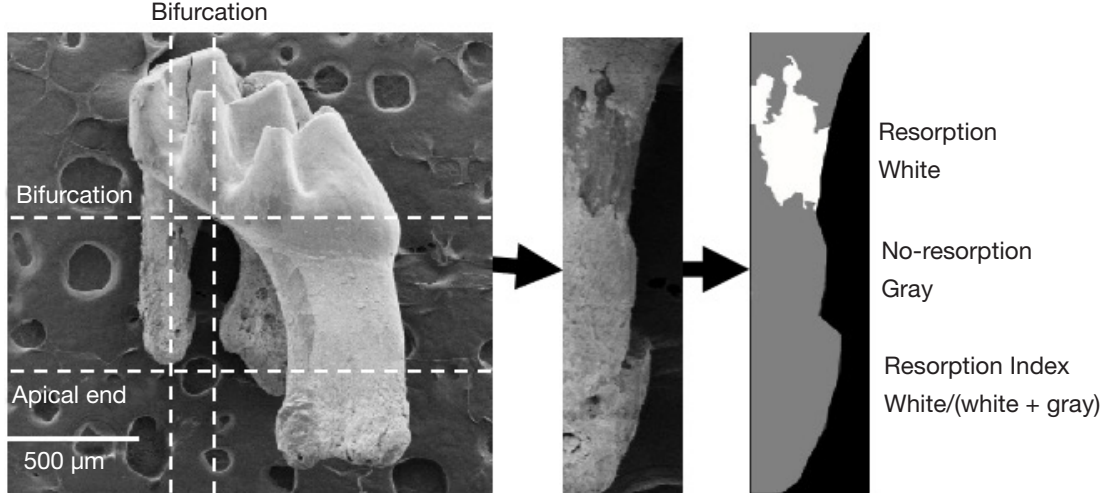

Figure 3 Effect of CSE on root resorption after orthodontic treatment. (A) Representative scanning electron micrographs of root resorption of the mesial surface of the distobuccal root of the maxillary first molar under orthodontic force, (a) control, $1 \mathrm{~W}$, (b) WT, $1 \mathrm{~W}$, (c) $\mathrm{CSE}^{-1}, 1 \mathrm{~W}$, (d) control, 2W, (e) WT, 2W, (f) $\mathrm{CSE}^{-/}, 2 \mathrm{~W}$, (g) control, $3 \mathrm{~W}$, (h) WT, $3 \mathrm{~W}$, (i) $\mathrm{CSE}^{-/-}, 3 \mathrm{~W}$ group; (B) arrows represent the direction of the teeth; bar $=500 \mu \mathrm{m}$; (C) the pattern diagram shows the absorption region (white) compared to the total root area (white + gray). The resorption proportion was calculated by dividing the resorbed area by the total root surface area [resorption proportion = white area/(white and grey area)]; (D) the graph shows the root resorption proportion in all the experimental groups. *, $\mathrm{P}<0.05$ versus the WT group; **, $\mathrm{P}<0.01$ versus the WT group. CSE, cystathionine gamma lyase; WT, wild type; M, mesial; D, distal; B, buccal; P, palatal; C, control; W, week.

extraction treatment are associated with orthodontically induced root resorption (24). In the clinical orthodontic practice, orthodontists attempt to find an "optimal" force to remove the dislocated tooth. However, root resorption seems to be inevitable even with light force (25). A previous study has found that $35 \mathrm{~g}$ of orthodontic force can effectively move orthodontic teeth while minimizing tissue damage (26). However, we found that using $35 \mathrm{~g}$ of force to move a mouse's 

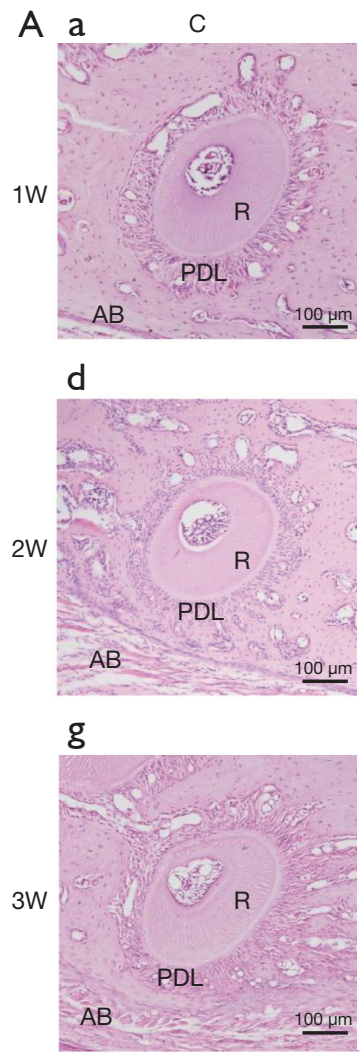
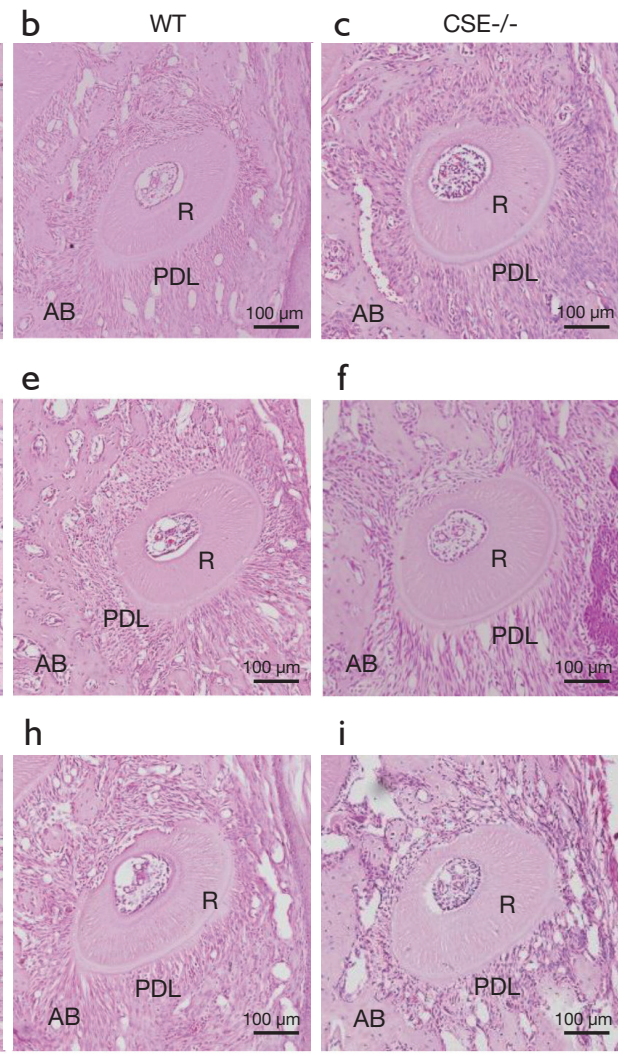

f

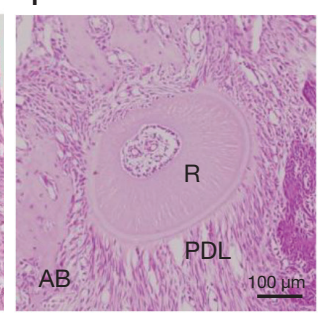

i

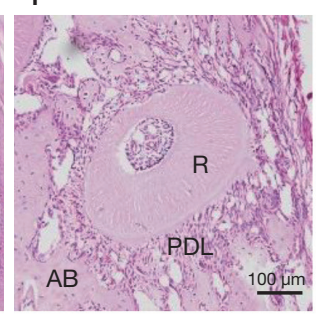

B
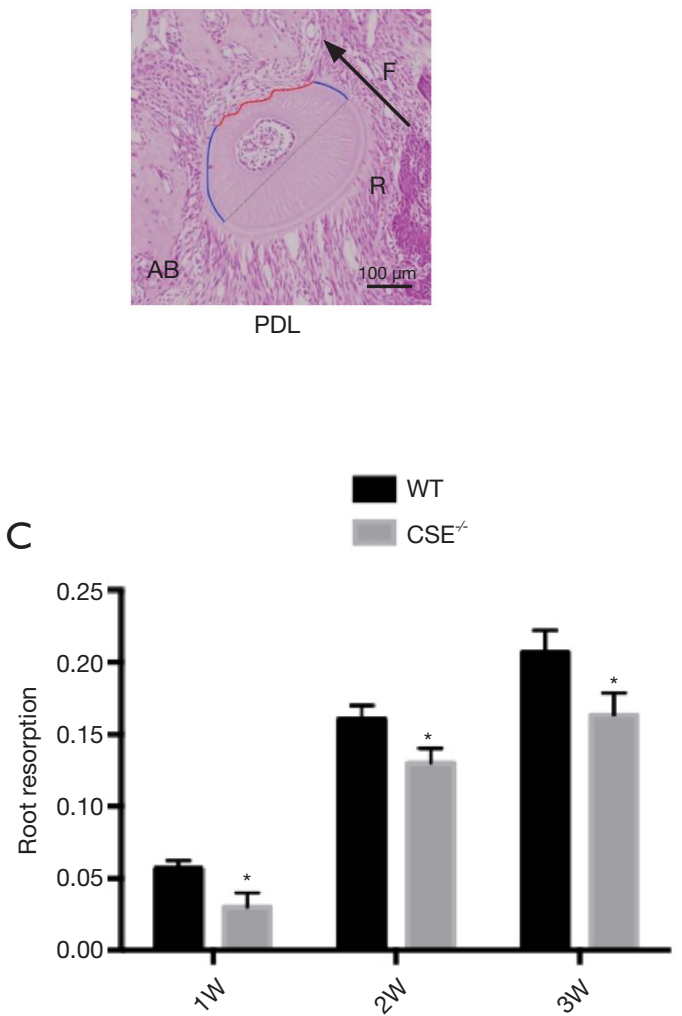

Figure 4 The morphological observation of root resorption in an orthodontic tooth movement model. (A) Effect of CSE on root resorption in an orthodontic tooth movement model observed by HE staining on horizontal histologic sections; (a) control, 1W, (b) WT, 1W, (c) $\mathrm{CSE}^{-/-}, 1 \mathrm{~W}$, (d) control, 2W, (e) WT, 2W, (f) $\mathrm{CSE}^{-/-}, 2 \mathrm{~W}$, (g) control, 3W, (h) WT, 3W, (i) CSE , $^{-/}$, $3 \mathrm{~W}$ group; (B) diagram indicating the resorbed line of the crater (red) compared with the pressure side of the root (red + blue). The resorption area ratio was calculated by dividing the resorbed area by the pressure side of the root [resorption area ratio $=$ red/(red + blue)]; $(\mathrm{C})$ graph demonstrating the ratio of the root resorption line in all experimental groups. *, $\mathrm{P}<0.05$ versus the WT group. F, orthodontic force; R, root; AB, alveolar bone; PDL, periodontal ligament; CSE, cystathionine gamma lyase; WT, wild type; C, control; W, week.

maxillary first molar for a week resulted in root resorption.

During orthodontic treatment, the mechanical force causes periodontal ligament cells to secrete RANKL and $O P G$, affecting differentiation and activation of osteoclasts and odontoclasts, and leading to root resorption (9-11,27). RANKL/OPG is an important inducer of osteoclastogenesis. Activation of OPG blocks osteoclastogenesis and accelerates mature osteoclast apoptosis by inhibiting RANK bing to RANK (28). RANK and OPG mRNA expression increased in tissue during heavy force-induced root resorption (29). Our results showed that CSE deficiency reduced orthodontically induced osteoclasts, RANKL/OPG mRNA expression in surrounding alveolar of the maxillary first molar and root resorption. The number of osteoclasts is correlated with the degree of root resorption (data not shown). The data indicate that CSE may mediate root resorption by affecting odontoclasts. Growth hormone promoted heavy force-induced root resorption by up-regulating RANKL/ OPG and IGF-1 in PDL cells at the early stage of tooth movement (14). OPG is osteoprotegerin that competitively binds to the RANK receptor RANKL site and inhibits the binding of RANK to RANKL, thereby inhibiting osteoclast activation (30). We examined the level of RANKL/OPG in surrounding periodontal tissue of the maxillary first molar in mice following 2-week orthodontic treatment. It was found that the number of osteoclast, the RANKL/OPG mRNA expression and root resorption in $\mathrm{CSE}^{-/}$mice was lower than that in WT mice. Our previous studies have shown that orthodontic force treated $\mathrm{CSE}^{-/-}$mouse had 
A a

WT

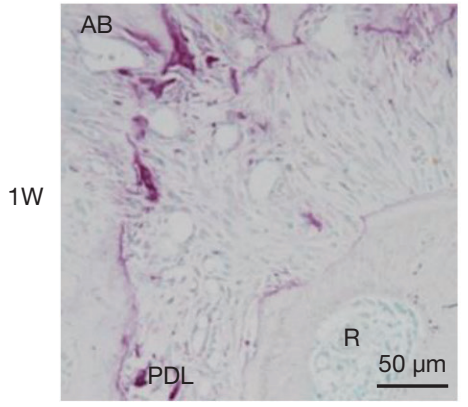

b

CSE
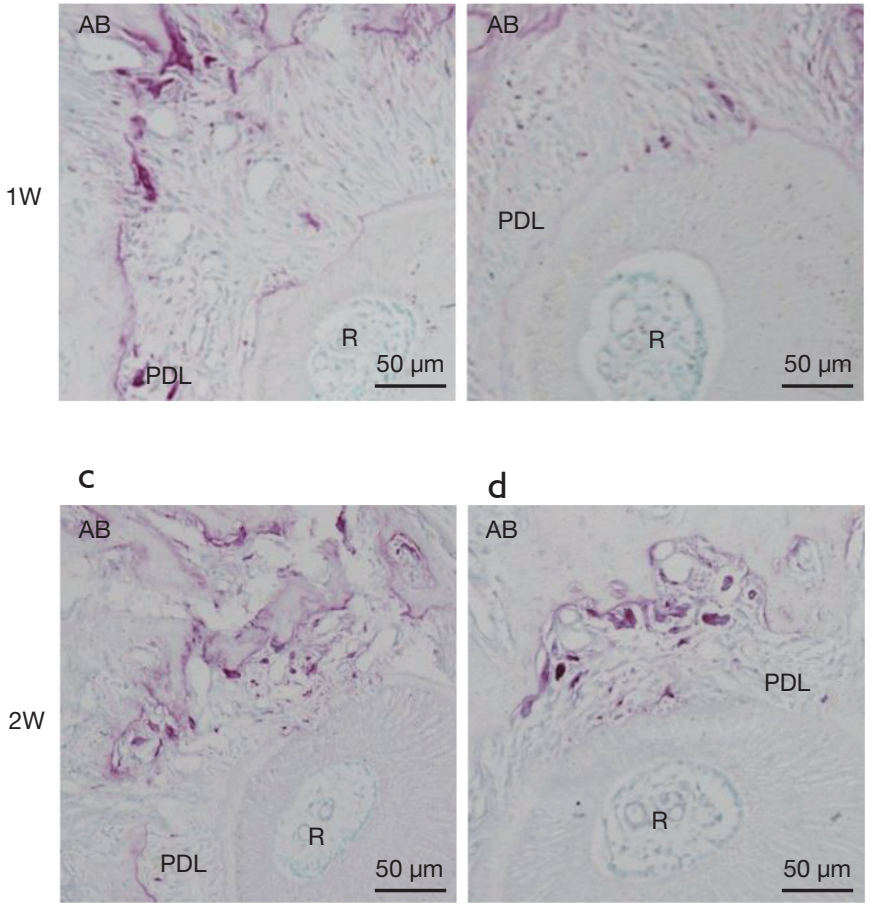

D

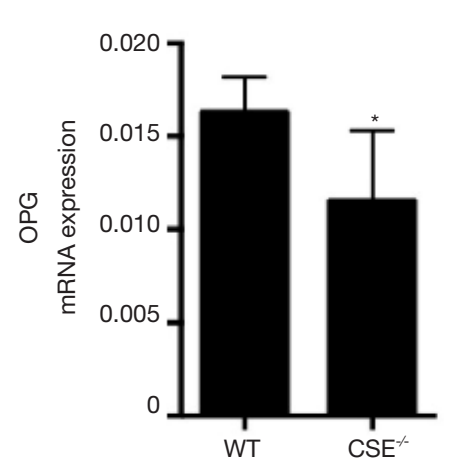

E

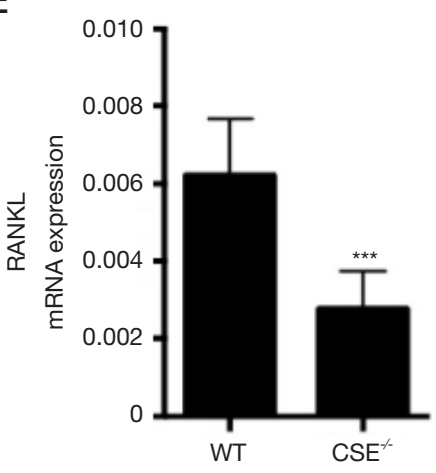

B

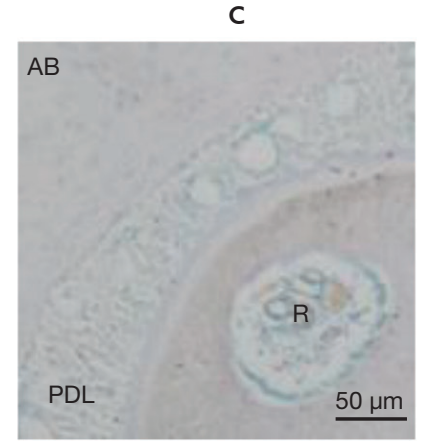

C

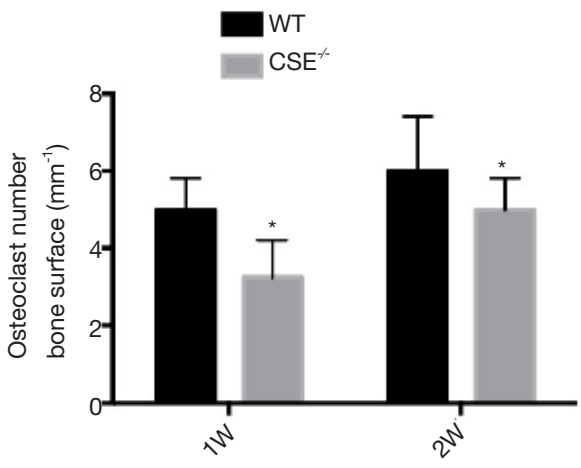

$\mathrm{F}$

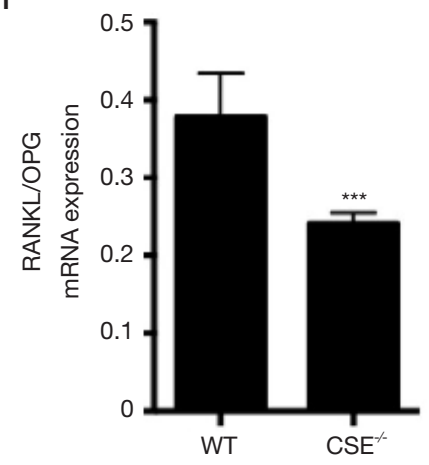

Figure 5 Odontoclasts and RANKL/OPG after orthodontic treatment for 2 weeks. (A) TRAP staining on horizontal histologic sections of the distobuccal root in (a) WT, $1 \mathrm{~W}$, (b) $\mathrm{CSE}^{-/}, 1 \mathrm{~W}$, (c) WT, $2 \mathrm{~W}$, (d) $\mathrm{CSE}^{-/-}, 2 \mathrm{~W}$; (B) Control groups; (C) the number of odontoclasts in mice; mRNA expression levels of (D) OPG, (E) RANKL and (F) RANKL/OPG in surrounding tissue of the maxillary first molar. *, P<0.05 versus the WT group; ***, $\mathrm{P}<0.005$ versus the WT group. CSE, cystathionine gamma lyase; WT, wild type; $\mathrm{R}$, root; AB, alveolar bone; PDL, periodontal ligament.

less osteoclasts compared with WT group (16). The data in this study suggested that CSE can promote orthodontic root resorption by increasing osteoclast differentiation and regulating RANK/RANKL/OPG (31). Odontoclasts and osteoclasts resorb tooth and alveolar bone, respectively. However, the process of bone resorption is similar (32).
Our study demonstrated that TRAP-positive cells existed not only on the root surface but also in the PDL tissues. Our results suggested that both osteoclasts and odontoclasts participated in the root resorption by a common mechanism, such as RANK/RANKL/OPG pathway or others (32). 


\section{Conclusions}

In conclusion, the results of our study demonstrated that CSE deficiency reduced formation of osteoclasts and odontoblasts, which consequently downregulated physiological and orthodontic root resorption by disruption of the mechanisms of RANKL/OPG-induced osteoclastogenesis and odontoclastogenesis, suggesting careful planning and execution of orthodontic mechanics, such as considering age at the start of treatment and duration of treatment.

\section{Acknowledgments}

Funding: This study was supported by the National Science Foundation of China (No. 81870791) and the Shanghai Science and Technology Committee (No. 17140903700).

\section{Footnote}

Conflicts of Interest: The authors have no conflicts of interest to declare.

Ethical Statement: The authors are accountable for all aspects of the work in ensuring that questions related to the accuracy or integrity of any part of the work are appropriately investigated and resolved. This study was approved by the Animal Use and Care Committee of Tongji University (2018-003).

\section{References}

1. Krishnan V, Davidovitch Z. On a path to unfolding the biological mechanisms of orthodontic tooth movement. J Dent Res 2009;88:597-608.

2. Henrikson T, Ekberg E, Nilner M. Can orthodontic treatment improve mastication? A controlled, prospective and longitudinal study. Swed Dent J 2009;33:59-65.

3. Kikuta J, Yamaguchi M, Shimizu M, et al. Notch signaling induces root resorption via RANKL and IL-6 from hPDL cells. J Dent Res 2015;94:140-7.

4. Weltman B, Vig KW, Fields HW, et al. Root resorption associated with orthodontic tooth movement: a systematic review. Am J Orthod Dentofacial Orthop 2010;137:46276; discussion 12A.

5. Roscoe MG, Meira JB, Cattaneo PM. Association of orthodontic force system and root resorption: A systematic review. Am J Orthod Dentofacial Orthop
2015;147:610-26.

6. Lossdörfer S, Götz W, Jäger A. Immunohistochemical localization of receptor activator of nuclear factor kappaB (RANK) and its ligand (RANKL) in human deciduous teeth. Calcif Tissue Int 2002;71:45-52.

7. Sahara N, Toyoki A, Ashizawa Y, et al. Cytodifferentiation of the odontoclast prior to the shedding of human deciduous teeth: an ultrastructural and cytochemical study. Anat Rec 1996;244:33-49.

8. Wise GE, King GJ. Mechanisms of tooth eruption and orthodontic tooth movement. J Dent Res 2008;87:414-34.

9. Kitaura H, Zhou P, Kim HJ, et al. M-CSF mediates TNF-induced inflammatory osteolysis. J Clin Invest 2005;115:3418-27.

10. Kitaura H, Yoshimatsu M, Fujimura Y, et al. An anti-cFms antibody inhibits orthodontic tooth movement. J Dent Res 2008;87:396-400.

11. Kitaura H, Fujimura $Y$, Yoshimatsu $M$, et al. An M-CSF receptor c-Fms antibody inhibits mechanical stressinduced root resorption during orthodontic tooth movement in mice. Angle Orthod 2009;79:835-41.

12. Yang PT, Kasai H, Xiao WG, et al. Increased expression of macrophage colony-stimulating factor in ankylosing spondylitis and rheumatoid arthritis. Ann Rheum Dis 2006;65:1671-2.

13. Nakano Y, Yamaguchi M, Fujita S, et al. Expressions of RANKL/RANK and M-CSF/c-fms in root resorption lacunae in rat molar by heavy orthodontic force. Eur J Orthod 2011;33:335-43.

14. Hu Y, Liu W, Liu Z, et al. Receptor activator of nuclear factor-kappa ligand, OPG, and IGF-I expression during orthodontically induced inflammatory root resorption in the recombinant human growth hormone-treated rats. Angle Orthod 2015;85:562-9.

15. $\mathrm{Pu} \mathrm{H}$, Hua Y. Hydrogen sulfide regulates bone remodeling and promotes orthodontic tooth movement. Mol Med Rep 2017;16:9415-22.

16. Mo S, Hua Y. Cystathionine gamma lyase-H2S contributes to osteoclastogenesis during bone remodeling induced by mechanical loading. Biochem Biophys Res Commun 2018;501:471-7.

17. Zheng Y, Liao F, Lin X, et al. Cystathionine gammaLyase-Hydrogen Sulfide Induces Runt-Related Transcription Factor 2 Sulfhydration, Thereby Increasing Osteoblast Activity to Promote Bone Fracture Healing. Antioxid Redox Signal 2017;27:742-53.

18. Irie K, Ekuni D, Yamamoto T, et al. A single application of hydrogen sulphide induces a transient osteoclast 
differentiation with RANKL expression in the rat model. Arch Oral Biol 2009;54:723-9.

19. Fujimura Y, Kitaura H, Yoshimatsu M, et al. Influence of bisphosphonates on orthodontic tooth movement in mice. Eur J Orthod 2009;31:572-7.

20. Pizzo G, Licata ME, Guiglia R, et al. Root resorption and orthodontic treatment. Review of the literature. Minerva Stomatol 2007;56:31-44.

21. Viecilli RF, Kar-Kuri MH, Varriale J, et al. Effects of initial stresses and time on orthodontic external root resorption. J Dent Res 2013;92:346-51.

22. Chung CJ, Soma K, Rittling SR, et al. OPN deficiency suppresses appearance of odontoclastic cells and resorption of the tooth root induced by experimental force application. J Cell Physiol 2008;214:614-20.

23. Krishnan V. Root Resorption with Orthodontic Mechanics: Pertinent Areas Revisited. Aust Dent J 2017;62 Suppl 1:71-7.

24. Samandara A, Papageorgiou SN, Ioannidou-Marathiotou I, et al. Evaluation of orthodontically induced external root resorption following orthodontic treatment using cone beam computed tomography (CBCT): a systematic review and meta-analysis. Eur J Orthod 2019;41:67-79.

25. Wishney $M$. Potential risks of orthodontic therapy: a critical review and conceptual framework. Aust Dent J 2017;62 Suppl 1:86-96.

Cite this article as: $\mathrm{Lu} \mathrm{C,} \mathrm{Chen} \mathrm{L,} \mathrm{Hua} \mathrm{Y.} \mathrm{Cystathionine}$ gamma lyase aggravates orthodontic root resorption in mice. Ann Transl Med 2019;7(23):787. doi: 10.21037/atm.2019.11.03
26. Taddei SR, Moura AP, Andrade I, Jr., et al. Experimental model of tooth movement in mice: a standardized protocol for studying bone remodeling under compression and tensile strains. J Biomech 2012;45:2729-35.

27. Matsuda Y, Motokawa M, Kaku M, et al. RANKL and OPG expression: Jiggling force affects root resorption in rats. Angle Orthod 2017;87:41-8.

28. Boyce BF, Xing L. Functions of RANKL/RANK/OPG in bone modeling and remodeling. Arch Biochem Biophys 2008;473:139-46.

29. Low E, Zoellner H, Kharbanda OP, et al. Expression of mRNA for osteoprotegerin and receptor activator of nuclear factor kappa beta ligand (RANKL) during root resorption induced by the application of heavy orthodontic forces on rat molars. Am J Orthod Dentofacial Orthop 2005;128:497-503.

30. Baud'huin M, Duplomb L, Teletchea S, et al. Osteoprotegerin: multiple partners for multiple functions. Cytokine Growth Factor Rev 2013;24:401-9.

31. Kurabayashi M. Hydrogen sulfide: a new regulator of osteoclastogenesis? Arterioscler Thromb Vasc Biol 2014;34:471-3.

32. Hakami Z, Kitaura H, Kimura K, et al. Effect of interleukin-4 on orthodontic tooth movement and associated root resorption. Eur J Orthod 2015;37:87-94. 